\title{
A Parasitic Resistance Extraction Method for Early Analysis of Complex Shaped Power Delivery Networks
}

\author{
K.K. Malinauskas \\ Silvaco Inc., kostas.malinauskas@silvaco.com
}

\begin{abstract}
This work presents a novel method of resistances extraction from arbitrary shaped 2D polygonal interconnects. It includes a new approach to resistor network construction based on medial axis transform and a simple set of analytical formulas for resistances estimation. The extracted circuits are compact enough while naturally mappable onto the original complex layout. These properties are useful in early design stage power delivery modeling with voltage drop or equivalent resistance maps visualization. The method is implemented within the industrial power integrity analysis system and demonstrated high efficiency while keeping enough accuracy.
\end{abstract}

Keywords - resistance extraction, power integrity, voltage drop, Voronoi diagram, medial axis.

\section{INTRODUCTION}

Ensuring the power integrity including IR-drop analysis is critical in the design of modern electronic devices [1][2]. After all, the circuit performance and reliability depend on the voltages distributed through the power supply networks, where complexity is constantly increasing. The problem is more severe when the supplied voltages become lower and the designs become larger and denser. Power requirements need to be considered throughout the whole design flow, since otherwise errors encountered late may require not just power re-routing but also significant redesign of the whole circuit.

Early design stage analysis helps to reveal potential problems that can be very costly and time consuming to correct later in the design process. Early IR-drop analysis tools should be fast but reasonably accurate, which is not an easy task, given the increasing scale of integrated circuits. The analysis results are expected to be visualized in the form of detailed voltage drop maps, allowing the circuit designer to easily localize critical layout areas.

Fast and reasonably accurate extraction of lumped resistor networks is essential in early IR-drop analysis. Their static and dynamic simulation implies solving the proportionally large linear systems. Therefore, to reduce the total analysis time, it is important that the extracted circuits be extremely compact. Taken together, the requirements for the extractor are difficult to fulfill if the power delivery networks have complex irregular layouts (non-uniform

* The original work first appeared in Russian language [0]. There was an error made in asymptotic resistance formula in Section III.C.1 ([0], p. 163), corrected in this paper. widths and directions, a variety of interconnect shapes), which may still be present in integrated circuits and printed circuit boards. There is a general trend of mask geometries simplification implied by modern VLSI lithography limitations. However multi-directional routing (usually in increments of $90^{\circ}, 60^{\circ}$, or $45^{\circ}$ [3]) can still be valid in upper metal layers and in old process nodes. Variable-width and bent power rails appear in systems on a chip when blocks with different layout styles are integrated. Analog circuit layouts with a large amount of manual design may have even more exotic interconnect shapes.

Known resistance extraction approaches are usually classified as either "exact" or heuristic [1][4]. The first ones numerically solve the electromagnetic field equations (finite difference, finite element, boundary element, Monte Carlo methods) [5]. The resulting extraction is highly accurate but requires fine meshing of complex shaped interconnects, leading to heavy computations and increased memory consumption, and thus is impractical for fast analysis of large circuits. It is common to reduce the extracted resistor networks, but their perfect mapping to the original layout can be lost [6]. Heuristic approaches apply analytical resistance formulas to predefined patterns found in the routing [7]-[9]. Their accuracy depends on the wire partitioning method, which can be non-trivial and ambiguous, plus their applicability is limited by the set of patterns (usually orthogonal).

In this paper, we propose a new heuristic method for parasitic resistance extraction from arbitrary shaped polygonal interconnects, based on the concept of the Voronoi diagram and medial axis transform. Geometric definitions are given in Section II. Section III presents our new method which is deterministic and constructs an optimal (linear) size lumped resistor network, perfectly mappable to the original layout. In Section IV the method applied to IRdrop analysis is discussed. Part V is devoted to the experimental results and further work directions.

\section{GEOMETRIC DEFINITIONS}

Voronoi diagram $\operatorname{VD}(S)$ of a set of planar objects $S$ is a subdivision of the plane into Voronoi cells $V R(s)$ - loci of points closer to an object $s \in S$ than to any other object [10]. VD is a planar graph, vertices of which are equidistant from three or more objects and edges are portions of bisectors generated by object pairs. 


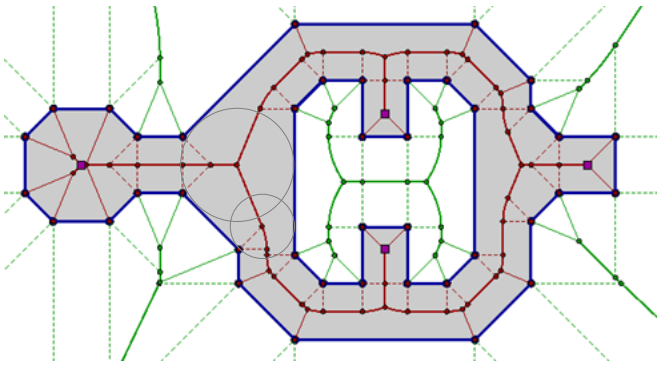

Fig. 1. Voronoi diagram and medial axis (internal solid lines) of a polygon

Voronoi diagram $\operatorname{VD}(P)$ of a simple polygon $P$ is a VD of its sides (line segments). To simplify VD construction each polygon side is represented as three objects - open line segment and its two endpoints [11]. Then in the plane with Euclidian metric every Voronoi edge is either rectilinear or parabolic. Bisectors of open segments and their endpoints produce secondary Voronoi edges (dashed lines in Fig. 1).

Medial axis of a simple polygon $P$ is a locus of centers of circles contained inside $P$ and touching its boundary at two or more points [11]. The circle radii define radius function along the medial axis. Medial axis together with its radius function is called medial axis transform (MAT). Medial axis of a polygon can be derived from Voronoi diagram by removing its external part and secondary edges (Fig. 1).

The above definitions are applicable to polygons with holes if their exterior and interior rings are simple polygons. For example, see Fig. 1, which depicts a polygon with a hole, its Voronoi diagram and medial axis.

\section{VORONOI DIAGRAM BASED RESISTANCE EXTRACTION METHOD}

\section{A. Basic idea}

Let us assume that all conductor geometries in each layer are merged in non-overlapping polygons (possibly with holes). This classical computational geometry problem can be efficiently solved e.g. using the plane sweep algorithm [10].

Note that in one-dimensional approximation the current flow between remote contacts roughly follows the conductor medial axis lines (compare Fig. 1 and Fig. 2). This observation underlies the proposed resistance extraction method, which includes the following steps for every polygonal conductor:

1. VD and MAT construction.

2. Terminal vertices assignment on the medial axis.

3. Conductor decomposition along the medial axis and resistor network construction.

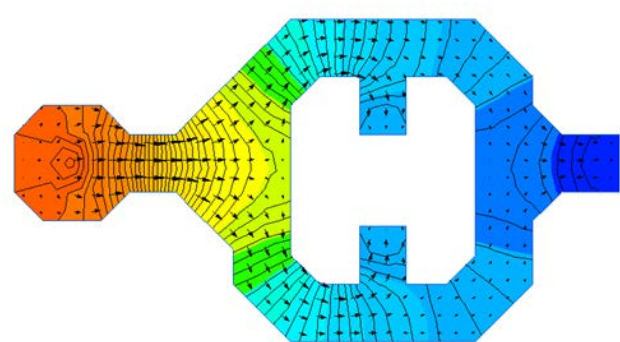

Fig. 2. Equipotentials and current flow in a complex-shaped conductor

\section{Resistance values calculation.}

$\mathrm{VD}$, and hence MAT, can be constructed in $O(n \log n)$ time and using $O(n)$ memory, where $n$ is the number of polygon sides. In particular, the plane sweep algorithm is widely used [10].

Next, let us assign terminal vertices at the medial axis points, closest to the contact pad (via interconnect) centers. If the closest point is internal to a medial axis edge, then the edge is split, and the new vertex becomes terminal.

\section{B. Conductor decomposition and resistor network con- struction}

Each medial axis edge $A B$ separates Voronoi cells of a pair of polygon sides $-s_{1}$ and $s_{2}$. Let $C_{1}, D_{1}, C_{2}, D_{2}$ denote the closest to $A$ and $B$ points on $s_{1}$ and $s_{2}$ respectively (see Fig. 3). Note that $C_{i}$ and $D_{i}$ are different if $s_{i}$ is a segment on a polygon boundary, and they coincide if $s_{i}$ is a concave vertex. Now in the general case we associate a hexagon $A C_{1} D_{1} B D_{2} C_{2}$ with every medial axis edge $A B$. Such primitive polygons are non-overlapping and form the partition of the original conductor (Fig. 3). In fact, their touching sides are typically orthogonal to the current flow near the conductor border. And the half-widths are equal to the radius function $\quad$ values: $\quad\left|A C_{1}\right|=\left|A C_{2}\right|=r(A)=r_{A}$, $\left|B D_{1}\right|=\left|B D_{2}\right|=r(B)=r_{B}$.

Equivalent resistor network is obtained naturally from the medial axis and the conductor decomposition (Fig. 4). Namely, the axis vertices and edges become the network nodes and lumped resistors. The approximate model assumes that the touching sides of adjacent primitive polygons are equipotential and serve as elementary resistor contacts. To prevent contact shorts the concave vertices of the original conductor are excluded from this model. The medial axis edges adjacent to the conductor boundary are removed from the network as having infinite resistances, and the corresponding primitive regions are considered equipotential. Note that the obtained resistor network, like the medial axis, has linear size: $O(n+m)$, where $n$ is the number of sides of the original conductor and $m$ is its terminal count. 


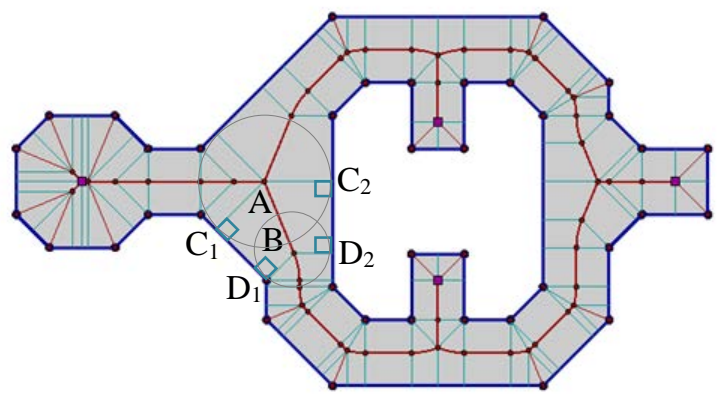

Fig. 3. Conductor decomposition along its medial axis

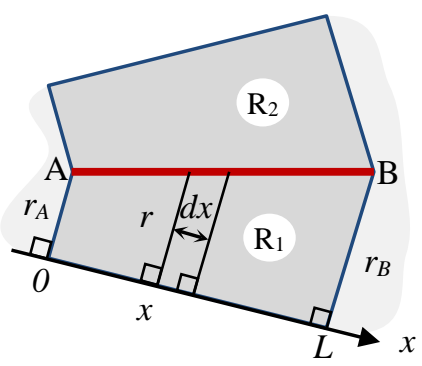

Fig. 5. Elementary resistor: two trapezoids

\section{Resistance values calculation}

Consider the elementary resistor generated by the medial axis edge $A B$ with radius function values $r_{A}$ and $r_{B}$ at its endpoints. The edge splits the resistor into two parts: $C_{1} D_{1} B A$ and $D_{2} C_{2} A B$ (Fig. 3). Three configurations are possible: 1) two trapezoids, 2) two triangles, 3) "trapezoid" and "triangle" with a common parabolic side. We will estimate the resistance of each configuration using the following assumptions:

a) the conductor is a homogeneous thin film with a sheet resistance of $1 \Omega$ /square (omitted in the formulas);

b) elementary resistor is equivalent to its two parts connected in parallel: $\mathrm{R}=R_{1} \| R_{2}=R_{1} R_{2} /\left(R_{1}+R_{2}\right)$.

\section{1) Two trapezoids}

Symmetric trapezoids are formed if the medial axis edge $A B$ is generated by two sides of the original polygon. Let us estimate the resistance by integrating along the $x$ axis passing through one of the sides (Fig. 5). The point $A$ projection specifies the origin $x=0$.

It follows from the similarity properties that the radius function varies linearly along the edge $A B$ :

$$
r=r_{A}+\frac{r_{B}-r_{A}}{L} x,
$$

where $L=\sqrt{|A \mathrm{~B}|^{2}-\left(r_{B}-r_{A}\right)^{2}}$ is the length of the edge $A B$ projection on the conductor boundary. Then

$$
d \mathrm{R}_{1}=\frac{d x}{r}=\frac{d x}{r_{A}+\frac{r_{\mathrm{B}}-r_{\mathrm{A}}}{L} x}=\frac{L \cdot d x}{\left(r_{\mathrm{B}}-r_{\mathrm{A}}\right) x+r_{A} L}
$$

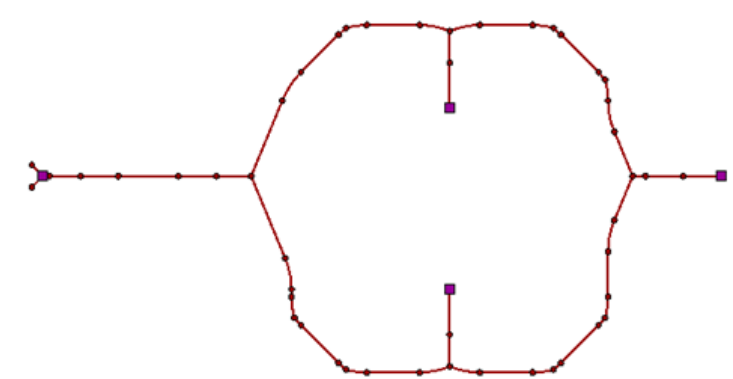

Fig. 4. Resistor network graph

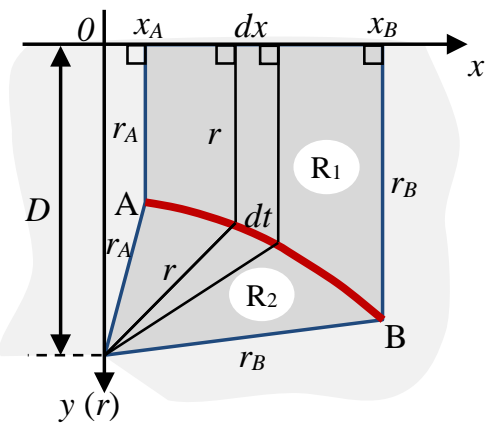

Fig. 7. Elementary resistor: "trapezoid" and "triangle"

$$
\begin{aligned}
\mathrm{R} & =\frac{\mathrm{R}_{1}}{2}=\frac{1}{2} \int_{0}^{L} \frac{L \cdot d x}{\left(r_{\mathrm{B}}-r_{\mathrm{A}}\right) x+r_{A} L}= \\
& =\left.\left(\frac{L \cdot \ln \left|\left(r_{\mathrm{B}}-r_{\mathrm{A}}\right) x+r_{A} L\right|}{2\left(r_{\mathrm{B}}-r_{\mathrm{A}}\right)}\right)\right|_{0} ^{\mathrm{L}}
\end{aligned}
$$$$
\begin{gathered}
\mathrm{R}=\frac{L \cdot \ln r_{B} / r_{A}}{2\left(r_{B}-r_{A}\right)} \text {, where } L=\sqrt{|A \mathrm{~B}|^{2}-\left(r_{B}-r_{A}\right)^{2}} \\
\mathrm{R}=\frac{L}{r_{A}+r_{B}} \text {, when } r_{A} \approx r_{B} .
\end{gathered}
$$

The last formula is asymptotic and solves the indeterminate form $0 / 0$ for the case $r_{A}=r_{B}$; it is also more accurate in practice when $r_{A}$ and $r_{B}$ values are close.

\section{2) Two triangles}

Two symmetrical triangles are formed if the medial axis edge $A B$ is generated by two concave vertices of the original polygon. We estimate the resistance by integrating along the $x$ axis passing through the edge, or along the polar angle $\alpha$ around the concave vertex (Fig. 6). The concave vertex projection specifies the origin $x=0(\alpha=0)$.

We first express the integration limits $x_{A, B}\left(\alpha_{A, B}\right)$ in terms of the known radius function values $r_{A, B}$ and the edge length $L=|A B|$. By the Pythagorean theorem, it follows that

$$
\left\{\begin{array}{l}
r_{A}^{2}=r_{0}^{2}+x_{A}^{2} \\
r_{B}^{2}=r_{0}^{2}+x_{B}^{2}
\end{array} \Rightarrow r_{B}^{2}-r_{A}^{2}=x_{B}^{2}-x_{A}{ }^{2}\right.
$$

Hence, given $L=x_{B}-x_{A}$, one can derive 
$x_{A, B}=\frac{r_{B}^{2}-r_{A}{ }^{2} \mp L^{2}}{2 L} ; \sin \alpha_{A, B}=\frac{x_{A, B}}{r_{A, B}}=\frac{r_{B}^{2}-r_{A}{ }^{2} \mp L^{2}}{2 L r_{A, B}}$.

Considering that $r=r_{0} / \cos \alpha$ and $x=r_{0} \tan \alpha$, we define the resistance differential:

$$
d \mathrm{R}_{1}=\frac{d x}{2 r}=\frac{r_{0} \cos \alpha \cdot d \alpha}{2 r_{0} \cos ^{2} \alpha}=\frac{d \alpha}{2 \cos \alpha} .
$$

Consequently,

$$
\begin{gathered}
\mathrm{R}=\mathrm{R}_{1} / 2=\frac{1}{2} \int_{\alpha_{A}}^{\alpha_{B}} \frac{d \alpha}{2 \cos \alpha}=\left.\left(\frac{1}{8} \ln \left|\frac{\sin \alpha+1}{\sin \alpha-1}\right|\right)\right|_{\alpha_{A}} ^{\alpha_{B}} . \\
R=\frac{1}{8} \ln \left|\frac{\left(r_{B}^{2}-r_{A}^{2}+L^{2}+2 L r_{B}\right)\left(r_{B}^{2}-r_{A}^{2}-L^{2}-2 L r_{A}\right)}{\left(r_{B}{ }^{2}-r_{A}^{2}+L^{2}-2 L r_{B}\right)\left(r_{B}{ }^{2}-r_{A}{ }^{2}-L^{2}+2 L r_{A}\right)}\right| \\
\text { where } L=|A \mathrm{~B}| .
\end{gathered}
$$

3) "Trapezoid" and "triangle"

The configuration arises if the medial axis edge $A B$ is parabolic and generated by original polygon side and concave vertex. We evaluate the resistance by integrating along the $x$ axis passing through the side of the polygon (Fig. 7). The concave vertex (parabola focus) projection defines the origin $x=0$, the edge endpoint projections the integration limits $x_{A}$ and $x_{B}$; the $y$ axis is directed to the concave vertex. Note that $y=r$ (radius function value). Having the polygon coordinates, it is possible to compute the distance $D$ from the concave vertex to the $x$ axis (doubled focal length of the parabola).

It follows from the parabola equation that the radius function varies quadratically along the edge $A B$ :

$$
r=\left(x^{2}+D^{2}\right) / 2 D ; \mathrm{d} r=(x \cdot \mathrm{d} x) / \mathrm{D} .
$$

Let us evaluate the "trapezoid” resistance:

$$
\mathrm{R}_{1}=\int_{x_{A}}^{x_{B}} \frac{d x}{r}=\int_{x_{A}}^{x_{B}} \frac{2 D \cdot d x}{x^{2}+D^{2}}=\left.\left(2 \tan ^{-1} \frac{x}{D}\right)\right|_{x_{A}} ^{x_{B}} .
$$

Given that $\mathrm{d} t=\sqrt{(d x)^{2}+(d r)^{2}}=\sqrt{x^{2}+D^{2}} d x / D$, let us evaluate the "triangle" resistance:

$$
\mathrm{R}_{2}=\int_{x_{A}}^{x_{B}} \frac{d t}{2 r}=\int_{x_{A}}^{x_{B}} \frac{d x}{\sqrt{x^{2}+D^{2}}}=\left.\left(\sinh ^{-1} \frac{x}{D}\right)\right|_{x_{A}} ^{x_{\mathrm{B}}} .
$$

Finally,

$$
\begin{gathered}
\mathrm{R}=2\left[\tan ^{-1} \frac{x_{B}}{D}-\tan ^{-1} \frac{x_{A}}{D}\right] \|\left[\sinh ^{-1} \frac{x_{B}}{D}-\sinh ^{-1} \frac{x_{A}}{D}\right] \\
\text { where } D-\text { doubled focal length of the parabola. }
\end{gathered}
$$

\section{IR-DROP ANALYSIS OF THE EXTRACTED NETWORKS}

We emphasize the following advantages of our extraction method when applied in early analysis of power sup- ply networks. First, the extracted circuits are sufficiently compact, since no fine meshes are used for the conductor decomposition. This allows us to substantially speed up the numerical linear solvers and to reduce memory consumption. Second, our method preserves the exact geometric mapping of the extracted resistor network onto the original layout, which helps the designer to visually analyze voltage drops and to localize critical areas. Indeed, there is a one-to-one mapping between the extracted resistors and the primitive polygons forming the conductor partition.

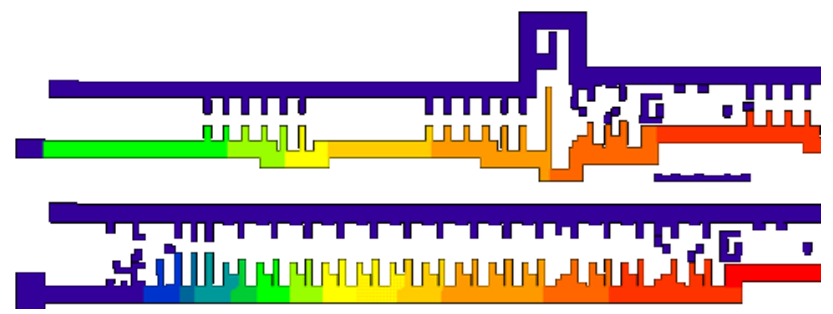

Fig. 8. Example of the voltage drop map region

The IR-drop map coloring is consistent with the equipotentials in our model (Fig. 8). The touching sides of decomposition primitive polygons are equipotential, their colors are determined by the voltage drops at the extracted circuit nodes. The gradient is applied to the primitive polygon inner regions in such a way that the isochromatic lines connect the medial axis edge inner points with the closest points on the conductor border. Along the edge, the color is determined by a linear interpolation of the voltage drop between the nodes. For ease of computation, parabolic edges can be straightened. In the same way, a map of equivalent resistances from voltage sources can be colored.

\section{RESULTS}

The proposed resistance extraction method is implemented in an industrial tool Silvaco Invar ${ }^{\circledR}$ Prime [12]. It is applied in early power integrity analysis of a broad range of designs including analogue and mixed signal VLSI, sensors, high-power circuits and flat panel displays. The extractor was tested within the flow performing analysis and visualization of voltage drops, as well as the equivalent resistances from voltage sources.

Experimental results proved the method advantages in comparison with the previously tested orthogonal tiling heuristics [1], generalized to polygonal conductors. As can be seen from the Table 1, on a test circuit our method allowed to reduce the extracted circuit size and the total simulation time by an order of magnitude, as well as to significantly reduce the memory consumption.

Table 1

\section{Efficiency comparison on a test circuit with a complex shaped layout}

\begin{tabular}{|l|r|r|}
\hline & $\begin{array}{c}\text { Tiling decom- } \\
\text { position [1] }\end{array}$ & $\begin{array}{c}\text { Decomposition based } \\
\text { on medial axis }\end{array}$ \\
\hline Nodes & 471533 & $110149(-77 \%)$ \\
Resistors & 880323 & $127360(-86 \%)$ \\
IR-drop analysis runtime & $163 \mathrm{sec}$ & $17 \mathrm{sec}(-90 \%)$ \\
Memory consumption & $1,27 \mathrm{~GB}$ & $0,6 \mathrm{~GB}(-40 \%)$ \\
\hline
\end{tabular}


Currently the author is further examining the new extraction method accuracy. It is difficult to do a detailed comparison of circuits extracted by different methods, because they may have non-matching topologies. However, visual comparison of equivalent resistance maps on realworld circuits showed generally quite a good correlation with the results of orthogonal tiling method (also heuristic). Locally, however, up to 5-15\% errors were observed. In future enhancement work it is planned to do a detailed error analysis and to further refine the elementary resistance formulas based on the results of accurate extraction using field solvers.

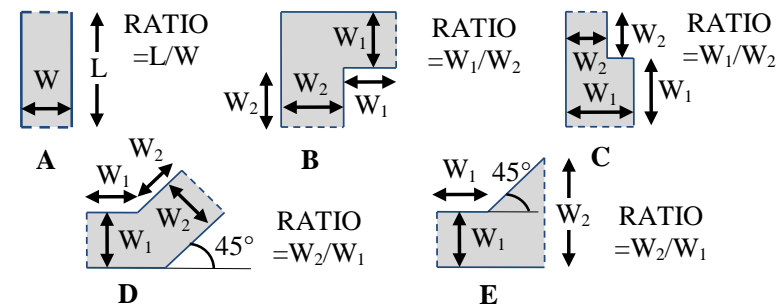

Fig. 9. Test conductor shapes [8]

At this point, the method is tested on a set of test conductors (Fig. 9) with known exact resistance values [8]. The observed error is below $10 \%$ as can be seen from Table 2. Note that if we extend the straight branches of the bent conductors B-E, e.g. 10 times, the total resistance error reduces by an order of magnitude.

Table 2

Extraction error on test conductor shapes

\begin{tabular}{|l|l|l|l|r|}
\hline Test & Ratio & $\mathbf{R}_{\text {exact }}[\mathbf{8}]$ & $\mathbf{R}_{\text {extracted }}$ & Error \\
\hline A & 1 & 1 & 1 & $0 \%$ \\
A & 5 & 5 & 5 & $0 \%$ \\
\hline B & 1 & 2.5 & 2.53 & $1 \%$ \\
B & 1.5 & 2.55 & 2.54 & $0 \%$ \\
B & 2 & 2.6 & 2.56 & $-2 \%$ \\
B & 3 & 2.75 & 2.6 & $-5 \%$ \\
\hline C & 1.5 & 2.1 & 1.96 & $-7 \%$ \\
C & 2 & 2.25 & 2.07 & $-8 \%$ \\
C & 3 & 2.5 & 2.27 & $-9 \%$ \\
C & 4 & 2.65 & 2.41 & $-9 \%$ \\
\hline D & 1 & 2.2 & 2.26 & $3 \%$ \\
D & 1.5 & 2.3 & 2.3 & $0 \%$ \\
D & 2 & 2.3 & 2.42 & $5 \%$ \\
D & 3 & 2.6 & 2.67 & $3 \%$ \\
\hline E & 1.5 & 1.45 & 1.34 & $-8 \%$ \\
E & 2 & 1.8 & 1.68 & $-7 \%$ \\
E & 3 & 2.3 & 2.17 & $-6 \%$ \\
E & 4 & 2.65 & 2.52 & $-5 \%$ \\
\hline
\end{tabular}

\section{CONCLUSION}

We developed a unique method for fast extraction of compact equivalent resistor networks from complex shaped thin film interconnects. Its application area is power integrity analysis of analog and mixed signal VLSI, as well as printed circuit boards and other electronic devices with irregular interconnect layout. The method accuracy is enough for its use in the early physical design stages, when the main requirement is a high speed of analysis and the ability to rapidly assess the quality of multiple layout design options.

\section{ACKNOWLEDGMENTS}

The author is grateful to Silvaco EDA engineers A.F. Samoilov, V.V. Davydov and V.N. Martynchik for valuable comments and practical recommendations, as well as to A.M. Marchenko (Mentor Graphics), who once motivated the author to study Voronoi diagrams and their applications in computer-aided design.

\section{REFERENCES}

[0] Malinauskas K.K. Metod ehkstrakcii parazitnyh soprotivlenij dlya rannego analiza cepej pitaniya s neregulyarnoj topologiej. MES-2018. Sb. trudov - Proc of MES-2018 conference. Moscow, 2018, pp. 161-166 (in Russian).

[1] Stark D. Analysis of Power Supply Networks in VLSI Circuits. Ph.D. Dissertation. Stanford University, Stanford, CA, USA, 1991

[2] Moroz Ya.N. Osobennosti metodov proektirovaniya sistem pitaniya vysokoproizvoditel'nyh mikroprocessorov na etape fizicheskogo proektirovaniya kristalla - Peculiarities in methods of designing power supply systems in highperformance microprocessors at the stage of physical design of the crystal. MES-2012. Sb. trudov. - Proc. of MES-2012 conference, Moscow, 2012, pp. 501-506 (in Russian).

[3] Kahng A.B., Lienig J., Markov I.L., Hu J. VLSI Physical Design: From Graph Partitioning to Timing Closure. Springer, Dordrecht, 2011.

[4] Bezrukov A.E., Rusakov A.S., Tkachev D.F., Hapaev M.M. Metody ekstrakcii parazitnyh elementov $\mathrm{v}$ integral'nyh shemah - IC parasitic elements extraction methods. MES2005. Sb. trudov. - Proc. of MES-2005 conference. Moscow, 2005, pp. 45-50 (in Russian).

[5] Yu W., Wang X. Advanced Field-Solver Techniques for RC Extraction of Integrated Circuits (1st ed.). Tsinghua University Press, Beijing and Springer-Verlag Berlin Heidelberg, 2016.

[6] Sheehan B.N. Realizable Reduction of RC Networks. IEEE Transactions on Computer-Aided Design of Integrated Circuits and Systems. 2007, v. 26, No. 8, pp. 1393-1407.

[7] Hall P.M. Resistance Calculations for Thin Film Patterns. Thin Solid Films. 1967/68, v. 1, No. 5, pp. 277-295.

[8] Horowitz M. Dutton R.W. Resistance Extraction from Mask Layout Data. IEEE Transactions on Computer-Aided Design of Integrated Circuits and Systems. 1983, v. 2, No. 3, pp. $145-150$.

[9] Spataro V. Counting Squares: A Method to Quickly Estimate PWB Trace Resistance. EDN Network, 2013. URL: https://www.edn.com (access date: 01.02.2018).

[10] Berg M., Cheong O., Kreveld M., Overmars M. Computational Geometry: Algorithms and Applications (3rd ed. ed.). TELOS, Santa Clara, CA, USA, 2008.

[11] Lee D.T. Medial Axis Transformation of a Planar Shape. IEEE Transactions on Pattern Analysis and Machine Intelligence. 1982, v. PAMI-4, No. 4, pp. 363-369.

[12] InVar Power - EM/IR - Thermal. URL: https://www.silvaco.com/products/analog_mixed_signal/in Var/invar.html (access date: 01.02.2018). 